University of Nebraska - Lincoln

DigitalCommons@University of Nebraska - Lincoln

Robert Katz Publications

Research Papers in Physics and Astronomy

January 1965

\title{
Width of Ion and Monopole Tracks in Emulsion
}

Robert Katz

University of Nebraska-Lincoln, rkatz2@unl.edu

J. J. Butts

Kansas State University, Manhattan, Kansas

Follow this and additional works at: https://digitalcommons.unl.edu/physicskatz

Part of the Physics Commons

Katz, Robert and Butts, J. J., "Width of Ion and Monopole Tracks in Emulsion" (1965). Robert Katz Publications. 25.

https://digitalcommons.unl.edu/physicskatz/25

This Article is brought to you for free and open access by the Research Papers in Physics and Astronomy at DigitalCommons@University of Nebraska - Lincoln. It has been accepted for inclusion in Robert Katz Publications by an authorized administrator of DigitalCommons@University of Nebraska - Lincoln. 
Physical Review 137:1B (January 11, 1965), pp. B198-B203.

\title{
Width of Ion and Monopole Tracks in Emulsion
}

\author{
Robert Katz and J. J. Butts \\ Kansas State University, Manhattan, Kansas
}

Submitted 13 July 1964; revised 14 September 1964

The width of the tracks of ions in emulsion has been calculated from the assumption that a developable image is formed when the energy dosage deposited by delta rays exceeds a threshold value, here found to be $6000 \mathrm{ergs} / \mathrm{cm}^{3}$ in $G-5$ emulsion. The theory agrees with measurements of track width obtained by projecting track images to a magnification of $3000 \times$ and tracing around their outline, while truncating isolated delta rays at their bases. Agreement is to within a grain diameter to a range of about $4 \mathrm{~cm}$. From the theory we infer that there is no $Z$ intelligence contained in the last $10 \mu$ of track length, and that very poor resolution in $Z$ (above 15) is obtained in thin-down region (last $150 \mu$ ). The calculation has been extended to infer the width of the track of a Dirac monopole as a function of its range. The length of track required for discrimination between ions and monopoles depends on the monopole mass. Thus monopoles of mass $5 \mathrm{amu}$, unit pole strength (137e / 2), and energy $1500 \mathrm{MeV}$ will have a range of $1000 \mu$ and can be confused in width with ions of charge 20 . On the other hand 3-amu unit-strength monopoles of $100-\mathrm{MeV}$ energy will have a range of $100 \mu$ and can be clearly distinguished from any ion. If $1 \mathrm{~cm}$ of track is available, any monopole (to mass $50 \mathrm{amu}$ ) can be clearly distinguished from all ions by the fact that its track width does not diminish with increasing range, and achieves a value of about $4 \mu$, in G5 emulsion.

C1965 The American Physical Society. Used by permission.

Online at URL: http://link.aps.org/abstract/PR/v137/pB198

DOI: 10.1103/PhysRev.137.B198 


\title{
Width of Ion and Monopole Tracks in Emulsion
}

\author{
Robert Katz and J. J. Butts \\ Kansas State University, Manhattan, Kansas \\ (Received 13 July 1964; revised manuscript received 14 September 1964)
}

\begin{abstract}
The width of the tracks of ions in emulsion has been calculated from the assumption that a developable image is formed when the energy dosage deposited by delta rays exceeds a threshold value, here found to be $6000 \mathrm{ergs} / \mathrm{cm}^{3}$ in $\mathrm{G}-5$ emulsion. The theory agrees with measurements of track width obtained by projecting track images to a magnification of $3000 \times$ and tracing around their outline, while truncating isolated delta rays at their bases. Agreement is to within a grain diameter to a range of about $4 \mathrm{~cm}$. From the theory we infer that there is no $Z$ intelligence contained in the last $10 \mu$ of track length, and that very poor resolution in $Z$ (above 15) is obtained in the thin-down region (last $150 \mu$ ). The calculation has been extended to infer the width of the track of a Dirac monopole as a function of its range. The length of track required for discrimination between ions and monopoles depends on the monopole mass. Thus monopoles of mass $5 \mathrm{amu}$, unit pole strength $(137 e / 2)$, and energy $1500 \mathrm{MeV}$ will have a range of $1000 \mu$, and can be confused in width with ions of charge 20. On the other hand 3-amu unit-strength monopoles of $100-\mathrm{MeV}$ energy will have a range of $100 \mu$ and can be clearly distinguished from any ion. If $1 \mathrm{~cm}$ of track is available, any monopole (to mass $50 \mathrm{amu}$ ) can be clearly distinguished from all ions by the fact that its track width does not diminish with increasing range, and achieves a value of about $4 \mu$, in G-5 emulsion.
\end{abstract}

\section{INTRODUCTION}

W HEN the tracks of heavy ions were first detected in emulsion exposed to cosmic rays, in 1948, their width was thought to be proportional to ionization, and the wedge-shaped appearance of the tracks near the end of the range was attributed to electron pickup.

In 1953 Lonchamp $^{1}$ projected machine-accelerated ions into emulsion and found that it was necessary to clarify the distinction between ionization and width, for the width was decreasing at ranges where the ionization was increasing. Electron pickup occurred much too near the end of the range to be responsible for the wedge-shaped appearance of the tracks. He proposed a simple theory in which width was calculated as the diameter of a cylinder, centered on the ion's path, through whose surface 400 delta rays passed in each $100 \mu$ of track length. By use of the delta-ray distribution formula, a range-energy relation for slow electrons, and an assumption of normal ejection of delta rays, a numerical value of track width could be computed. Though Lonchamp identified the mechanism of width formation, his formulation was not quantitative.

A substantial improvement in Lonchamp's theory was made by Bizzeti and Della Corte, ${ }^{2}$ in 1959 , by altering the track formation criterion from one of delta-ray flux to one of energy flux. At low ion energies, in the thin-down region, where delta rays emerging from the critical cylinder had a residual range less than a grain diameter, this theory was in reasonable agreement with experiment, but our extrapolation of the theory to higher ion energies yielded theoretical values for the track width which did not fit experimental data.

The present theory of track formation is built on the foundation of the work of Bizzeti and Della Corte. It assumes that charged particles transfer energy to a

${ }^{1}$ J. P. Lonchamp, J. Phys. Radium 14, 433 (1953).

2 P. G. Bizzeti and M. Della Corte, Nuovo Cimento 11, 317 (1959). medium through the formation of delta rays, and that the detector is sensitized when these delta rays deposit energy in the medium at a rate exceeding a threshold dosage. In the case of emulsion, all photographic grains touching the cylinder of critical diameter are assumed to be sensitized. The track width is then the sum of the diameter of the critical cylinder, the diameter of a developed grain, and the diameter of an undeveloped grain (see Fig. 1). Processing is assumed to affect the width through the diameter of the developed grain, and possibly through the value of the threshold dosage.

Most detectors lack both the spatial resolution and the sensitivity for these detailed considerations of the mechanism of track formation to be significant. But, since electron-sensitive emulsion has both sensitivity and spatial resolution, the secondary ionization from delta rays in the tracks of heavy ions dominates the appearance of these tracks.

In the present calculation delta rays are assumed to be ejected with effective spherical symmetry, to obey a power-law "diffusion-length" energy relation, and to have an energy spectrum in accordance with the usual delta-ray distribution formula. We are forced to treat the coefficient of the power law as an adjustable parameter, for want of complete data. Similarly, the threshold dosage for emulsion sensitization is treated as an

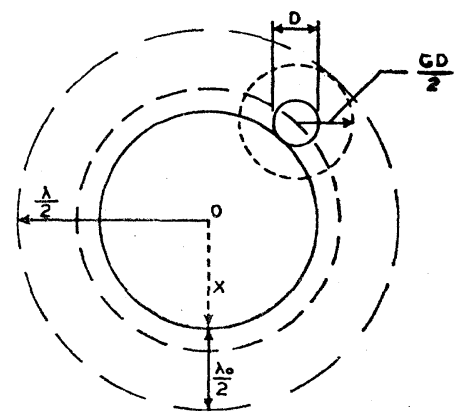

Fig. 1. Cross section of track. A grain of diameter $D$ tangent to the critical cylinder grows in development to diameter $G D$. The total track width after development is $\lambda=2 x+(G+1) D$ 
adjustable parameter. The numerical values of these parameters are determined from the over-all fit of track-width data with computed curves.

Studies of the identification of the Dirac monopole in emulsion, based on the track-width theory of Lonchamp, were initiated by Katz and Parnell ${ }^{3}$ in 1959. According to this theory the tracks of heavy ions are thin at high energy. As the energy diminishes, the tracks increase in width, passing through a maximum, and then thin down in the last several hundred microns of range. The tracks of monopoles are to be wedge shaped, being wide at high energy and thinning down continuously as they approach the end of their range without passing through a maximum. While the qualitative description of the difference between ion and monopole tracks remains intact, the quantitative details of the description of the width of ion tracks has been significantly improved in the present work, with corresponding improvement to be expected in the extrapolation to the track of a monopole. The track of a unitpole-strength Dirac monopole will achieve and retain a width slightly greater than $4 \mu$ in $G-5$ emulsion. This width will be achieved at ranges of approximately $70 A \mu$, where $A$ is the monopole mass in amu. The saturation width itself depends on the pole strength. Thus, any track whose width never decreases as its range increases may be identified as a monopole. The mass may be determined from the range at which the width reaches its saturation value, and the pole strength may be determined from the magnitude of this width.

Machine experiments to manufacture monopoles ${ }^{4,5}$ have thus far been limited to possible masses of $3 \mathrm{amu}$. Monopoles of this mass could be readily identified, in tracks $100 \mu$ long. For this range an energy of $100 \mathrm{MeV}$ is required. This energy would be attained by a Dirac monopole in $5 \mathrm{~cm}$ in a field of $1 \mathrm{kOe}$.

\section{THEORY OF TRACK WIDTH}

The track width may be calculated by postulating that an undeveloped grain will be sensitized if any part of its volume is sufficiently close to the path of the primary particle to receive the threshold dosage for latent image formation $E^{*}$. From symmetry, all grains included in and tangent to a cylinder of radius $x$ will be sensitized. On processing, the outermost grains will grow from diameter $D$ to a new diameter $G D$, so that an examination of the geometry of Fig. 1 leads to the result that the track width $\lambda$ is given by the equation

where

$$
\lambda=2 x+\lambda_{0},
$$

$$
\lambda_{0}=(G+1) D .
$$

\footnotetext{
${ }^{3}$ R. Katz and D. R. Parnell, Phys. Rev. 116, 236 (1959).

${ }^{4}$ E. Amaldi, G. Baroni, H. Bradner, H. G. deCarvalho, L. Hoffman, A. Manfredini, and G. Vanderhaeghe, CERN 63-13, Geneva, 1963 (unpublished).

${ }^{5}$ E. M. Purcell, G. B. Collins, T. Fujii, J. Hornbostel, and F. Turkot. Phys. Rev. 129, 2326 (1963).
}

At low ion energies where the range of delta rays is significantly less than a grain diameter, the minimum width achieved is $\lambda_{0}$, the sum of the diameters of a developed grain and an undeveloped grain. The formulation here is consistent with our observation that the track width in the first $10 \mu$ is constant and is the same for all $Z$. We have observed that there are differences in $\lambda_{0}$ for tracks terminating at different depths in the emulsion, apparently due to processing.

If $E(x, \beta, Z)$ is the energy flux through a cylinder of radius $x$ carried by delta rays made by an ion charge $Z e$ and speed $\beta c$, then the energy density deposited in a shell of thickness $\Delta x$ is $(E(x, \beta, Z)-E(x+\Delta x, \beta, Z)) /(2 \pi x \Delta x)$. In the limit of small $\Delta x$ we require that this expression approach a limit $E^{*}$, an adjustable parameter, so that

$$
E^{*}=-\frac{1}{2 \pi x} \frac{\partial E(x, \beta, Z)}{\partial x}
$$

and we must turn to the task of calculating $E(x, \beta, Z)$.

Let $\bar{W}(x, w)$ represent the average energy carried through a cylinder of radius $x$ by an electron of energy $w$, the average being with respect to the direction of emission. The total energy flux through the cylinder of unit length, is obtained by integrating $\bar{W} d n$, where $d n$ is the number of delta rays per unit path length having energies between $w$ and $w+d w$, produced by an ion of effective charge $Z e$ moving with speed $\beta c$. We have

$$
d n=\frac{2 \pi N e^{4} Z^{2} d w}{m c^{2} \beta^{2} w^{2}}=C \frac{Z^{2}}{\beta^{2}} \frac{d w}{w^{2}},
$$

where the mass of an electron and its charge are $m$ and $e$, and $N$ is the number density of electrons in the medium (emulsion) through which the ion passes. The constant $C$ is implicitly defined in Eq. (4) as $C=2 \pi N e^{4} /\left(m c^{2}\right)$. We use observed trackwidths (corresponding to electrons of energy $w \lesssim 40 \mathrm{keV}$ ) to simplify the rigorous delta-ray distribution formula which contains an additional factor of $\left(1-\beta^{2} w / w_{\max }\right)$. This factor is essentially $\left(1-w / 2 m c^{2} \gamma^{2}\right)$, or nearly 1 , for this calculation. This equation must be supplemented by an expression for the effective charge $Z e$ of an ion of atomic number $Z^{\prime}$ at speed $\beta c$ in emulsion; according to $\operatorname{Barkas}^{6} Z e$ may be expressed as

$$
Z e=Z^{\prime} e\left[1-\exp \left(-125 \beta / Z^{\prime 2 / 3}\right)\right] .
$$

Using Eq. (4) we obtain

$$
\begin{aligned}
E(x, \beta, Z) & =\int_{w(x)}^{w_{\max }} \bar{W}(x, w) d n \\
& =C \frac{Z^{2}}{\beta^{2}} \int_{w(x)}^{w_{\max }} \frac{\bar{W}(x, w)}{w} \frac{d w}{w}
\end{aligned}
$$

${ }^{6} \mathrm{H}$. Barkas, Nuclear Research Emulsions (Academic Press Inc., New York, 1963), Vol. 1, Chap. 9, p. 371. 
where the lower limit $w(x)$, is the energy of a delta ray whose diffusion length is just sufficient to penetrate the cylinder if it is ejected normally, and the upper energy limit $w_{\max }=2 m c^{2} \beta^{2} \gamma^{2}$, is the maximum delta-ray energy as determined by kinematics.

To complete the calculation we need a diffusion relation for low-energy electrons. Range-energy relations for electrons customarily give the maximum range or the extrapolated range expected from a population of monoenergetic electrons. The present calculation requires an average range. Since no data are available for the diffusion length at these low energies, we have altered an experimental power-law range-energy relation by treating its coefficient $k$ as an adjustable parameter. We have

$$
r=k w^{\alpha},
$$

where $r$ is the diffusion length in microns, $w$ is the electron energy in $\mathrm{keV}$. The exponent is taken to be 1.72 , a value suggested by Glocker ${ }^{7}$ for energies between 1 and $100 \mathrm{keV}$.

For simplicity, delta rays are assumed to follow straight-line paths and their angular distribution is taken as effectively spherically symmetric. In the calculation a more relaxed angular distribution is actually used; that is,

$$
f(\theta)+f(\pi-\theta)=1 / 2 \pi .
$$

The sum of the fore and aft electron-ejection probabilities is constant.

Reexpressing Eq. (7) as $w=(r / k)^{1 / \alpha}$, and changing the variable in Eq. (6), we obtain

$$
E(x, \beta, Z)=\frac{C Z^{2}}{\alpha \beta^{2}} \int_{x}^{R} \frac{\bar{W}(x, r)}{w(r)} \frac{d r}{r},
$$

where the energy $w$ of the delta ray has been written as $w(\boldsymbol{r})$ for emphasis and where $R=k\left(2 m c^{2} \beta^{2} \gamma^{2}\right)^{\alpha}$ is the maximum diffusion length of an electron. Bizzeti and

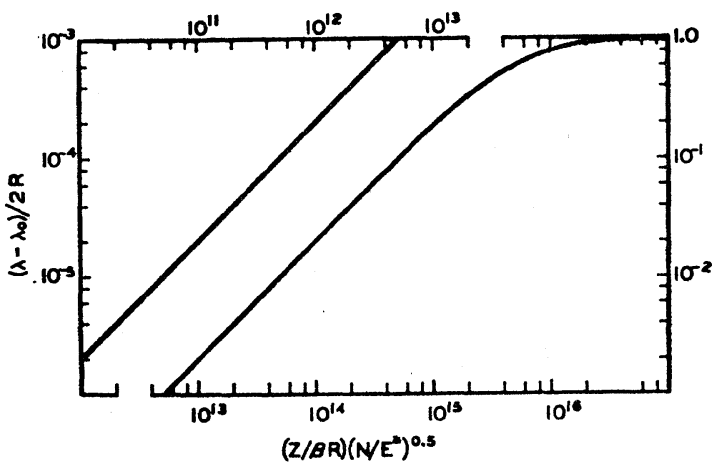

FIG. 2. Universal curve (Gaussian units) for calculating track width, $\lambda$, as a function of $\beta$ for ions of charge $Z e$. The electron density $N$, the threshold dosage $E^{*}$, the maximum diffusion length of an electron $R$, and the sum of the developed and undeveloped grain diameters $\lambda_{0}$, all depend upon the emulsion.

${ }^{7}$ R. Glocker, Z. Naturforsch. 3a, 129 (1948).

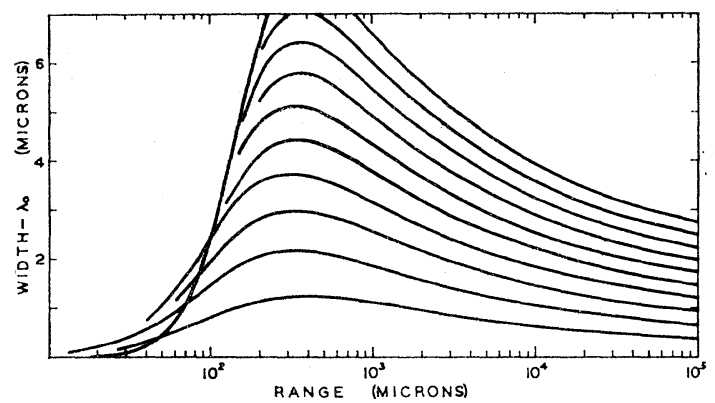

Fig. 3. Theoretical plot of reduced track width $\left(\lambda-\lambda_{0}\right)$ versus range for ions of $Z=5,10,15,20,25, \cdots, 50$. The constant $\lambda_{0}$ is equal to the sum of the developed and undeveloped grain diameters (approximately $0.75 \mu$ for $G-5$ emulsion). At large ranges the width increases with $Z$. The mass assignment is for the most abundant isotope.

Della Corte ${ }^{2}$ have shown that the integral in Eq. (9) is a function of the ratio $x / R$ only. Thus we have

$$
E(x, \beta, Z)=\frac{C}{\alpha} \frac{Z^{2}}{\beta^{2}} I\left(\frac{x}{R}\right) .
$$

The track width may now be determined by applying the condition of constant dosage from Eq. (3). On writing

$$
I^{\prime}\left(\frac{x}{r}\right)=\frac{\partial}{\partial x} I\left(\frac{x}{R}\right)
$$

replacing $C$ by its value and rearranging, we find

$$
\frac{Z}{\beta R}\left(\frac{N}{E^{*}}\right)^{1 / 2}=\left[\frac{-\alpha m c^{2}}{e^{4}} \frac{x}{R} \frac{1}{I^{\prime}(x / R)}\right]^{1 / 2} .
$$

We have used this equation to plot a universal curve for the calculation of track width in emulsion, or in any detector (see Fig. 2). For $G-5$ emulsion, $N=1.045 \times 10^{24}$ electrons $/ \mathrm{cm}^{3}$. For best fit with our track width data we have taken the threshold sensitivity of $G-5$ emulsion to electrons to be $6000 \mathrm{ergs} / \mathrm{cm}^{3}$. Since the maximum diffusion length $R$ is a function of $\beta$, as previously given, we may find $x$ from the curve for given values of $\beta$ and $Z$. To fit our data, $k$ [Eq. (7)] was adjusted to 0.006 .

Finally, accepted values of proton range $^{8}$ are used in conjunction with Heckman's formula ${ }^{9}$ to convert $\beta$ to range for heavy ions, taking electron pickup into account. The results are plotted in Fig. 3 .

\section{EXPERIMENT, AND ITS CONNECTION WITH THEORY}

The track width is subject to statistical fluctuations. To find an average width comparable to the computed width, we measure the area per unit length of a segment

${ }^{8}$ M. M. Shapiro, in Handbuch der Physik, edited by S. Flügge (Springer-Verlag, Berlin, 1958), Vol. 45, p. 366.

${ }^{9}$ H. H. Heckman, B. L. Perkins, W. G. Simon, F. M. Smith, and W. H. Barkas, Phys. Rev. 117, 544 (1960). 
of track which is long enough to smooth out the irregularities, but not so long that the average width changes appreciably in the segment. Ten-micron segments were used in the first $150 \mu$, and $50-\mu$ segments were used at greater ranges.

Measurements were made by tracing around a projected image of the track, magnified $3000 \times$ by a Leitz nuclear-emulsion microscope provided with a xenon lamp. Disconnected clumps of silver were ignored and isolated delta rays were cut off at their base. The areas of the profiles traced in this manner were measured with a planimeter.

To normalize the data for variation in processing (grain size) with depth, the width of a track of $3-\mathrm{cm}$ residual range was measured at different depths. Over small intervals at this large range, any width variation is due to variation of $\lambda_{0}$ with depth in the emulsion. Through use of these measurements, all data were normalized to a depth of $150 \mu$ by an additive correction.

The theoretical results indicate that the tracks of all ions have the same width $\lambda_{0}$ in the last $10 \mu$ of their range. This result is supported by our normalized data, and may be noted in published photographs. ${ }^{10} \mathrm{We}$ have found that $\lambda_{0}=0.75 \mu$, in our emulsions, a value consistent with average data for $G-5$ emulsion given by Barkas, ${ }^{6}$ who gives the diameter of a developed grain as $0.5 \mu$ and the diameter of an undeveloped grain as $0.27 \mu$.

The combination of parameters yielding the best fit to our data are $E^{*}=6000 \mathrm{ergs} / \mathrm{cm}^{3}$, and $k=0.006$ [see Eq. (7)]. Our determination of $k$ gives a diffusion length which is about one-third the maximum range of $10-\mathrm{keV}$ electrons, as given by Feldman. ${ }^{11}$

Width measurements were made on eight tracks found in emulsion exposed to cosmic rays at an altitude

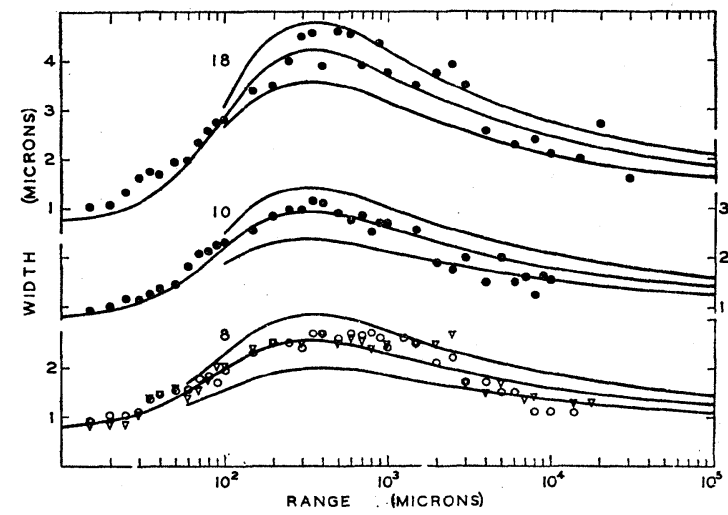

FIG. 4. Data for four tracks found in $G-5$ emulsion exposed to the cosmic rays. The families of curves represent the best fitting theoretical curve $(Z=8,8,10,18)$. To show $Z$ discrimination, each assigned $Z$ is bounded by $Z \pm Z^{1 / 2}$. All the data are normalized to an emulsion depth where $\lambda_{0}=0.75 \mu$.

${ }^{10}$ C. F. Powell, P. H. Fowler, and D. H. Perkins, A Study of Elementary Particles by the Photographic Method (Pergamon Press Inc., New York, 1959), pp. 170-175.

${ }^{11}$ C. Feldman, Phys. Rev. 117, 455 (1960).

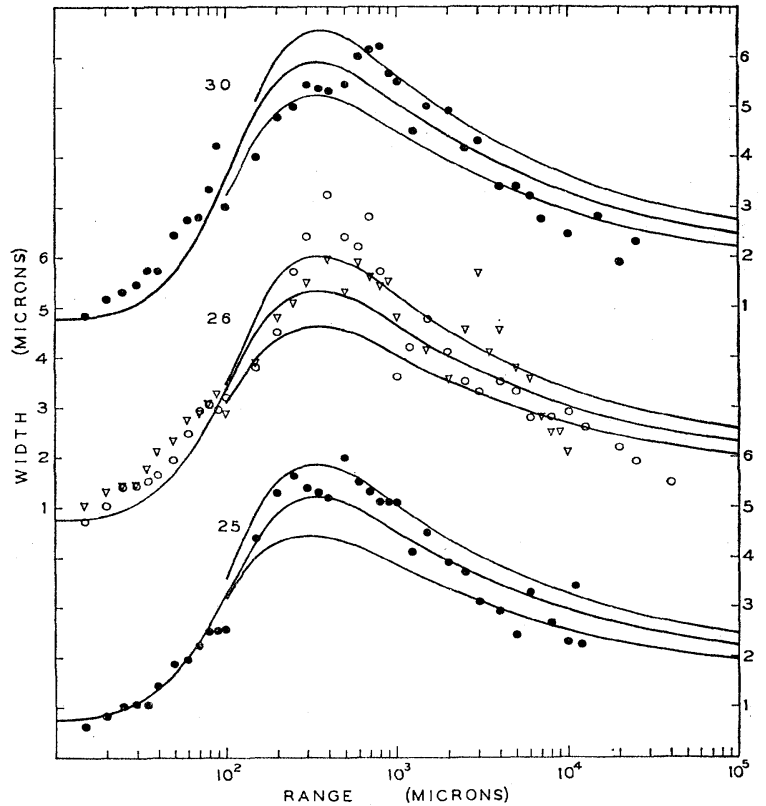

FIg. 5. Data for four tracks found in $G-5$ emulsion exposed to the cosmic rays. The families of curves represent the best fitting theoretical curve $(Z=25,26,26,30)$, each bounded by $Z \pm Z^{1 / 2}$, as in Fig. 4. All the data are normalized to an emulsion depth where $\lambda_{0}=0.75 \mu$.

of $100000 \mathrm{ft}$. The results are shown in Figs. 4 and 5, plotted together with the theoretical curves for the best estimate of $Z$, and for $Z \pm Z^{1 / 2}$ to show discrimination. Most of the measured tracks agree with the theory to within a grain diameter $(0.5 \mu)$ at all ranges to $4 \mathrm{~cm}$. In all calculations the ion mass was taken as that of the most abundant isotope.

Comparison of the theory with published data for the width of machine accelerated ions is shown in Figs. 6 and 7, where the published data of Bizzeti and Della Corte $^{2}$ and of Skjeggestad ${ }^{12}$ are plotted over our theoretical curves.

Data and theory show that the range at which the width of an ion track is a maximum is substantially independent of $Z$.

It appears that the best $Z$ discrimination should be obtained when measurements are made near the maximum width. The roughness of the track outline in this region is offset by the slower variation of width with range (as compared to the thin-down region of the track), allowing measurement of longer segments.

The theory predicts that the width-range curves of ions of different $Z$ cross in the thin-down region. For ranges less than $150 \mu$, a plot of reduced width $\left(\lambda-\lambda_{0}\right)$ versus $Z$ shows a maximum (see Fig. 8). This effect is easily understood in terms of the width limitation imposed by the maximum diffusion length of delta rays. In the thin-down region the very large number of delta rays per unit track length causes the theoretical

\footnotetext{
${ }^{12}$ O. Skjeggestad, Nuovo Cimento 8, 927 (1958).
} 

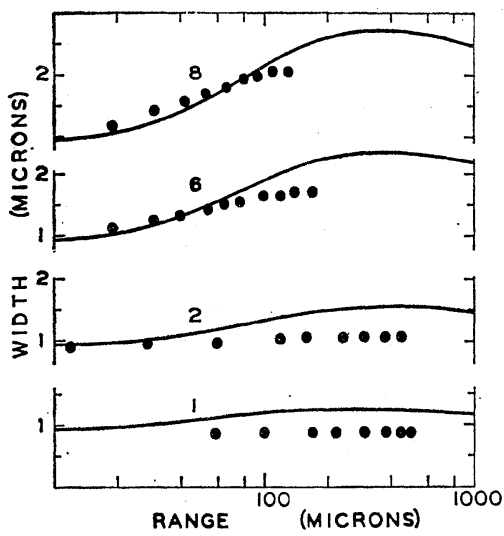

FIG. 6. Comparison of theory with published photometric track-width data of Bizzeti and Della Corte (see footnote 2) obtained with accelerated ions $(Z=1,2,6,8)$. $\lambda_{0}$ $=0.8 \mu$.

width to be approximately equal to the diffusion length of the electrons of maximum energy. Thus, in the thindown region the width is almost proportional to $\beta^{2 \alpha}$. Ac a range of, say, $50 \mu$, the speed of an ion of $Z=50$ is less than that of a $Z=20$ ion. This is due to electron pickup ${ }^{7}$ : $\mathrm{a}_{27} \mathrm{Co}_{59}$ ion has an extra range of $120 \mu$ and $a_{50} \mathrm{Sn}_{120}$ ion has an extra range of $360 \mu$ over the range otherwise predicted from proton ranges. The track of the lighter ion is therefore wider than the track of the heavier ion. These considerations imply that $Z$ assignments based on width, or on the area of the thin-down wedge, are insensitive to $Z$ and are ambiguous above $Z=15$. The situation is vastly improved at a range of $150 \mu$.

\section{WIDTH OF MONOPOLE TRACKS}

A slowly moving monopole of pole strength $g$ generates an electric field normal to the trajectory of magnitude $g v b / c r^{3}$, where $r$ is the distance to the field point, and $b$ is the impact parameter. We expect a monopole to interact with matter through this field. A slowly moving charge carries with it an electric field whose component normal to the trajectory is $Z e b / r^{3}$ (Gaussian units). A comparison of these fields suggests that

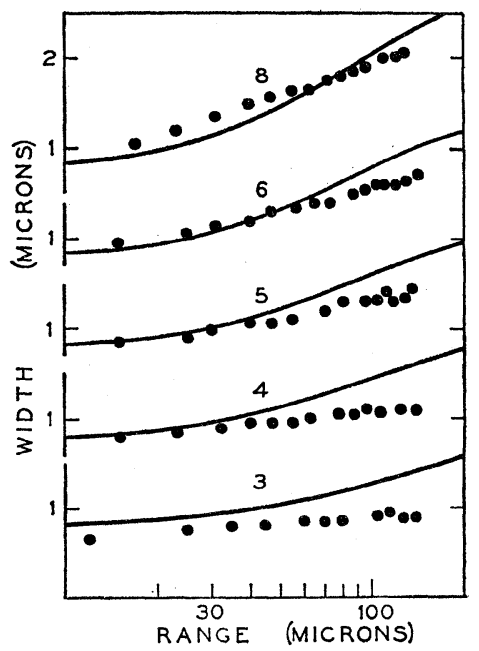

Fig. 7. Comparison of theory with published visual track-width data of Skeggestad (see footnote 9) obtained with accelerated ions $(Z=3,4,5,6,8)$. $\lambda_{0}=0.8 \mu$. formulas for ionization and delta-ray distribution for moving ions can be converted to formulas appropriate to monopoles by replacing $Z e$ by $g \beta$. This recipe was derived rigorously by Bauer ${ }^{13}$ and by Cole ${ }^{14}$ in 1951.

We may find an expression for the delta ray distribution for monopoles by making the substitution $g \beta$ for Ze in Eq. (4) to obtain

$$
d n=C\left(g^{2} / e^{2}\right)\left(d w / w^{2}\right) .
$$

The delta-ray distributions for charges and poles are plotted in Fig. 9. Note that the delta-ray distribution formula remains valid at high energies, because of compensating factors of $\gamma$ which appear at high speeds from the Lorentz contraction and time dilation. ${ }^{15}$ In Fig. 9, curve $A$ is the delta-ray distribution for a Dirac monopole (the kinematic cutoff depends on $\beta$ and is shown as a dashed line for $\beta=0.25$ at $W=68 \mathrm{keV})$. The other two curves show the variation in the delta-ray distribution with $\beta$ for ions of $Z=16$. It is significant that the delta-ray distribution for poles depends on $\beta$ only through the changing value of the kinematic cutoff.

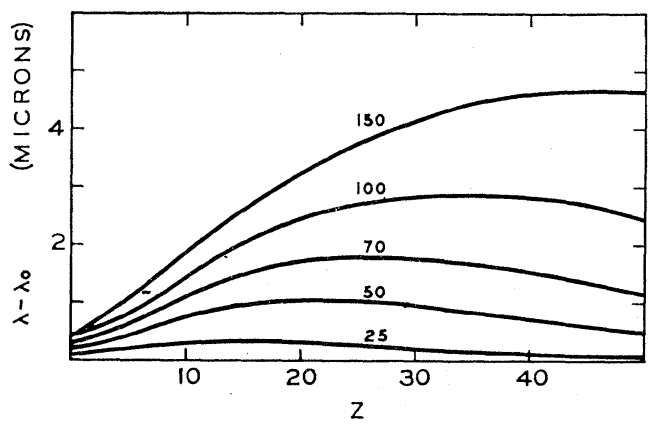

FIG. 8. Curves showing the theoretical variation of track width with $Z$ at ranges of $25,50,70,100$, and $150 \mu$. Notice, for example, that the widest tracks at $50 \mu$ are due to ions of charge $20 e$.

An increase in $\beta$ adds more delta rays of higher energy leaving the rest of the distribution unchanged. Thus, an increase in $\beta$ cannot produce a decrease in track width. The width of a monopole track must increase monotonically with $\beta$, independent of the detailed mechanism by which a track-width theory converts the delta-ray distribution to track width.

A universal curve for determining monopole track width as a function of $\beta$ may be obtained by making the substitution $g \beta=Z e$ in the abscissa label of Fig. 2 .

To establish a range $\beta$ relation for monopoles, the ionization formula of Bethe ${ }^{16}$ was integrated, making shell corrections according to the procedure of Vigneron. ${ }^{17}$ The resulting range values were found to be

${ }^{13}$ E. Bauer, Proc. Cambridge Phil. Soc. 47, 777 (1951).

${ }^{14}$ H. J. D. Cole, Proc. Cambridge Phil. Soc. 47, 196 (1951).

${ }^{16}$ B. Rossi, High Energy Particles (Prentice-Hall, Inc., New York, 1952), Chap. 2, p. 18.

${ }^{16}$ M. S. Livingston and H. A. Bethe, Rev. Mod. Phys. 9, 245 (1937).

${ }_{17}$ P. L. Vigneron, J. Phys. Radium 14, 145 (1953). 
in agreement with experimental data for protons of $\beta<0.9$ to within $5 \% .^{8}$ The ionization formula was then converted by the usual recipe $(Z e \rightarrow g \beta)$ and integrated to give range- $\beta$ values for monopoles. Our calculations of monopole range were in agreement with a curve of Amaldi et $a l .^{4}$ There is some question about the interaction of poles with the medium in the limit of low velocity, when the electric field of the moving pole drops to zero. We infer from arguments by Malkus ${ }^{18}$ and others that a pole will interact strongly with matter when its energy is of the order of tens of electron volts, and that the range we have calculated here is within a few microns or within $5 \%$ of the true range, whichever is greater.

The results of these calculations are plotted in Fig. 10, where the reduced width $\left(\lambda-\lambda_{0}\right)$ of the tracks of Dirac monopoles of unit strength $(137 e / 2)$ and of twice unit strength (137e) is plotted against a background of track-width-range curves for ions.

The most obvious feature of the monopole track is the absence of a maximum width, a feature which is required by the delta-ray distribution relation for poles, Eq. (12), independent of other aspects of the trackwidth theory. The track of a monopole is strongly dependent on its mass and its magnetic charge, as shown in Fig. 10. The range at which the track reaches saturation width is determined by the mass of the monopole, while the magnitude of the saturation width is determined by the pole strength.

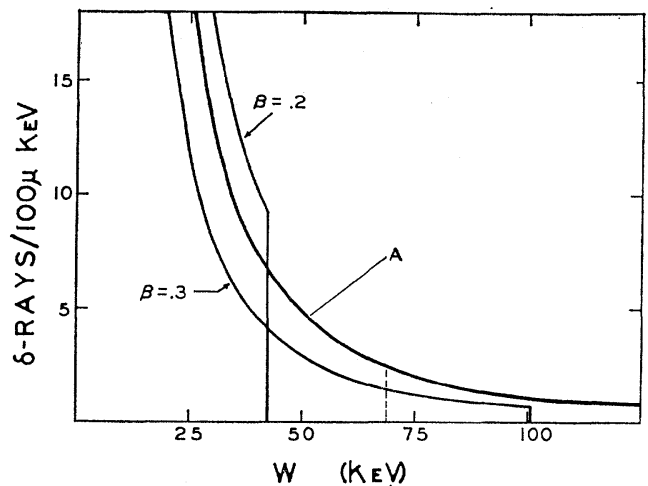

Fig. 9. The spectrum of delta rays in $G-5$ emulsion for unit Dirac monopoles (curve A), and for $Z=16$ ions at $\beta=0.2$ and 0.3 . The delta-ray spectrum for poles is the same for all $\beta$, except for the position of the kinematic cutoff, shown as a dashed line at $w=68 \mathrm{keV}$ for $\beta=0.25$.

\footnotetext{
${ }^{18}$ W. V. R. Malkus, Phys. Rev. 83, 899 (1951).
}

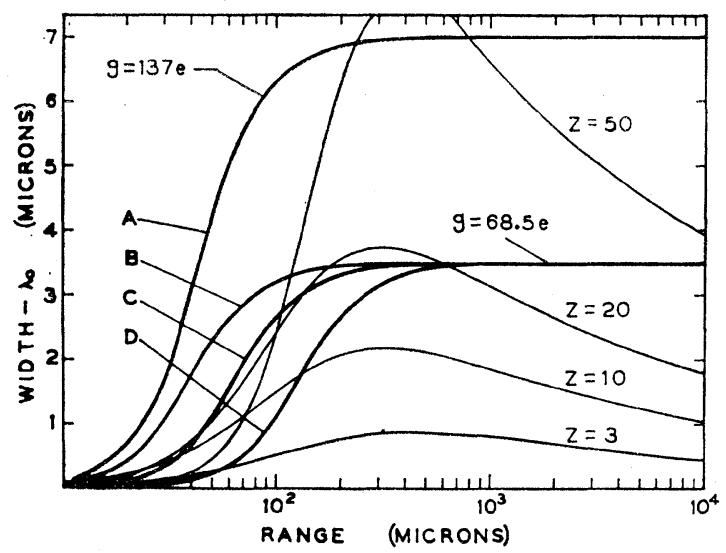

FIG. 10. Comparison of the width of tracks of ions $(Z=3,10$, $20,50)$ and monopoles of unit pole strength and twice unit pole strength in $G-5$ emulsion. The family of monopole curves (heavy lines) includes the anticipated track width for a monopole of strength $g=137 e$ and $m=10 \mathrm{amu}$ (curve A), and of $g=68.5 e$ with $m=3$ amu (curve B), $m=5$ amu (curve C), and $m=10 \mathrm{amu}$ (curve D).

The region of possible confusion with ions depends on the mass assumed for the monopole. For masses less than $3 \mathrm{amu}$, the largest possible mass obtainable in recent accelerator experiments, ${ }^{4,5}$ monopoles would be easily identified if only the last $50 \mu$ of track were available. Monopoles with mass greater than $10 \mathrm{amu}$ would be clearly distinguished from ions if $500 \mu$ of track were available for measurement. For other masses, a pole might be confused with an ion on the basis of trackwidth measurements to ranges of $1000 \mu$. For example, a 5 -amu pole and a $Z=20$ ion display nearly the same range-width relation to $1000 \mu$.

In a recent paper Goto ${ }^{19}$ has predicted that monopoles from cosmic space would be accelerated to energies of $10^{20} \mathrm{eV}$. Monopoles of these energies may be expected to display a constant track width of approximately $4 \mu$ in G-5 emulsion, according to these calculations, and should be readily identifiable.

\section{ACKNOWLEDGMENTS}

We are grateful to D. E. Guss for the emulsion used in these measurements. We are also indebted to E. W. Hoffman and M. R. Querry for their help in the calculations, and especially to E. J. Kobetich for his help in measurement and machine computation.

${ }^{19}$ E. Goto, Progr. Theoret. Phys. (Kyoto) 30, 700 (1963). 